\title{
$\beta$-catenin promotes endothelial survival by regulating eNOS activity and flow-dependent anti-apoptotic gene expression
}

Virginia Tajadura', Marie Haugsten Hansen', Joy Smith', Hannah Charles ${ }^{1}$, Matthew Rickman², Keith Farrell-Dillon?', Vasco Claro (1)', Christina Warboys ${ }^{2,3}$ and Albert Ferro (iD ${ }^{1}$

\begin{abstract}
Increased endothelial cell (EC) apoptosis is associated with the development of atherosclerotic plaques that develop predominantly at sites exposed to disturbed flow (DF). Strategies to promote EC survival may therefore represent a novel therapeutic approach in cardiovascular disease. Nitric oxide (NO) and $\beta$-catenin have both been shown to promote cell survival and they interact in ECs as we previously demonstrated. Here we investigated the physiological role of $\beta$-catenin as a mediator of NO-induced cell survival in ECs. We found that $\beta$-catenin depleted human umbilical vein ECs (HUVEC) stimulated with pharmacological activators of endothelial NO synthase (eNOS) showed a reduction in eNOS phosphorylation (Ser1177) as well as reduced intracellular cyclic guanosine monophosphate levels compared to control cells in static cultures. In addition, $\beta$-catenin depletion abrogated the protective effects of the NO donor, $\mathrm{S}$ nitroso-N-acetylpenicillamine, during TNFa- and $\mathrm{H}_{2} \mathrm{O}_{2}$-induced apoptosis. Using an orbital shaker to generate shear stress, we confirmed eNOS and $\beta$-catenin interaction in HUVEC exposed to undisturbed flow and DF and showed that $\beta$-catenin depletion reduced eNOS phosphorylation. $\beta$-catenin depletion promoted apoptosis exclusively in HUVEC exposed to DF as did inhibition of soluble guanylate cyclase (sGC) or $\beta$-catenin transcriptional activity. The expression of the pro-survival genes, $\mathrm{BCl}-2$ and survivin was also reduced following inhibition of $\beta$-catenin transcriptional activity, as was the expression of eNOS. In conclusion, our data demonstrate that $\beta$-catenin is a positive regulator of eNOS activity and cell survival in human ECs. SGC activity and $\beta$-catenin-dependent transcription of BCl-2, survivin, BIRC3 and eNOS are essential to maintain cell survival in ECs under DF.
\end{abstract}

\section{Introduction}

Apoptosis is implicated in a number of cardiovascular diseases, in particular the development and progression of atherosclerosis, plaque rupture, ischaemia reperfusion injury and heart failure ${ }^{1}$. Increased endothelial cell (EC) apoptosis is associated with the development of atherosclerotic plaques ${ }^{2}$, vascular injury ${ }^{3}$ and raised vessel

\footnotetext{
Correspondence: Christina Warboys (cwarboys@rvc.ac.uk) or

Albert Ferro (albert.ferro@kcl.ac.uk)

${ }^{1}$ School of Cardiovascular Medicine \& Sciences, British Heart Foundation

Centre of Research Excellence, King's College London, London SE1 9NH, UK

${ }^{2}$ Department of Bioengineering, Imperial College London, London SW7 2BP,

UK

Full list of author information is available at the end of the article.

Edited by A. Finazzi-Agrò
}

permeability ${ }^{4}$. Strategies to promote cell survival may therefore be important in reducing cardiovascular disease.

Atherosclerosis is characterised by dysfunction of the vascular endothelium which is associated with reduced bioavailability and bioactivity of nitric oxide $(\mathrm{NO})^{5}$. Endothelial NO synthase (eNOS), the main vascular source of NO, confers protection from cardiovascular disease $^{6}$, through a number of mechanisms including inhibition of EC apoptosis ${ }^{7-11}$. Similarly, laminar shear stress prevents TNF $\alpha$ and $\mathrm{H}_{2} \mathrm{O}_{2}$-induced apoptosis and this effect is dependent on eNOS activity ${ }^{9}$. NO has previously been shown to inhibit apoptosis at several levels; via S-nitrosylation of caspases ${ }^{8,12}$, increased expression and stability of Bcl-2 by destabilization of MKP-3 
mRNA $^{13}$ and inhibition of JNK signalling pathways ${ }^{7,14}$. Recently we identified $\beta$-catenin as a novel eNOS binding partner in human umbilical vein EC (HUVEC) and showed that pharmacological activation of eNOS, acting through soluble guanylate cyclase (sGC) and cyclic guanosine monophosphate (cGMP), promotes nuclear translocation of $\beta$-catenin and transcription of some $\beta$-catenin target genes providing evidence of a novel signalling axis in $\mathrm{EC}^{15}$.

$\beta$-catenin is a component of the adherens junction, linking VE-cadherin to the actin cytoskeleton, where it plays an important role in the dynamic regulation of endothelial permeability ${ }^{16}$. Non-junctional (cytosolic) $\beta$-catenin is rapidly degraded under resting conditions by interacting with an inhibitory complex comprised of APC, axin and GSK3 $\beta$ that phosphorylates $\beta$-catenin and targets it for ubiquitin-mediated degradation ${ }^{16}$. Cytosolic $\beta$-catenin can be stabilised by canonical and noncanonical Wnt signalling pathways and, as recently established, by NO-cGMP signalling ${ }^{15}$, whereby dephosphorylated (active) $\beta$-catenin accumulates and translocates to the nucleus where it regulates gene expression through its interaction with TCF-LEF transcription factors ${ }^{16}$. A subset of $\beta$-catenin target genes is associated with anti-apoptotic (cell survival) functions raising the possibility that NO may promote cell survival through transcriptional activation of $\beta$-catenin.

Here we sought to assess the role of $\beta$-catenin in eNOS signalling and the physiological role of the NO-cGMP$\beta$-catenin axis on apoptosis in EC under disturbed (atheroprone) or undisturbed (atheroprotective) flow.

\section{Materials and methods}

\section{Cell culture, transfection and application of shear stress}

HUVEC were purchased from Promocell as pools from several donors and cultured in Promocell Endothelial Cell Growth Medium (containing 2\% FCS). Cells were cultured at $37^{\circ} \mathrm{C}$ and $5 \% \mathrm{CO}_{2}$ and confluent HUVEC at up to passage 6 were used for experiments. Human aortic ECs (HAEC) from single donors were purchased from Promocell and cultured on fibronectin in Endothelial Growth Medium MV (Promocell). HAEC were used up to passage 6. Mouse pulmonary ECs (MPEC) were cultured as previously described ${ }^{15}$. Culture media was supplemented with $100 \mathrm{U} / \mathrm{ml}$ penicillin and $100 \mu \mathrm{g} / \mathrm{ml}$ streptomycin. Antibiotic free media was used for the cell assays and added before application of flow (see below) and/or before a treatment was added. At least three different biologically independent cell batches were studied for each experiment.

RNA interference was performed using siRNA sequences specific for human target genes. Non-targeting scrambled sequences were used as a control. HUVEC were transfected with siRNAs $(100 \mathrm{nM})$ using
Lipofectamine RNAiMAX (Invitrogen) in serum-free media (Optimem, Invitrogen) without antibiotics. Medium was changed to growth medium $16 \mathrm{~h}$ after transfection. Scrambled control and $\beta$-catenin siRNA pool were purchased from Ambion and Santa Cruz, respectively.

For assays in which application of flow was required, an orbital shaker was used. EC were seeded at passages 3-7 onto fibronectin-coated six-well plates. Where experiments required immunostaining, EC were seeded onto fibronectin-coated glass-bottom plates (In Vitro Scientific). Once monolayers were confluent (after 24-48 h), media was changed and the six-well plates were placed onto an orbital rotating platform (Grant Instruments) housed inside the incubator and cultured for a further $72 \mathrm{~h}$. The radius of orbit of the orbital shaker was $10 \mathrm{~mm}$ and the rotation rate was set to $150 \mathrm{rpm}$, which caused swirling of the culture medium over the cell surface that creates distinct flow patterns at the centre and edge of the well; cells at the edge are exposed to undisturbed uniaxial flow (UF), whereas cells at the centre are exposed to disturbed multidirectional flow (DF) ${ }^{17}$.

For transfection of HUVEC for flow experiments, immediately prior to seeding into six-well plates, HUVEC were transfected with $100 \mathrm{nM} \mathrm{MISSION}^{\circledR}$ pre-designed and validated siRNA targeting $\beta$-catenin (Sigma) or $100 \mathrm{nM}$ scrambled control (Ambion) by electroporation. Electroporation was carried out using a Neon ${ }^{\mathrm{TM}}$ Transfection System according to manufacturer's instructions. Cells were seeded at a density of $1 \times 10^{5}$ cells per well and cultured under static conditions for $\sim 6 \mathrm{~h}$ to allow cells to adhere and become confluent before exposure to flow using the orbital shaker method. For analysis of protein expression, three wells were pooled for each flow condition. Knockdown of target proteins was confirmed by western blot for each experiment.

EC were exposed to disturbed or UF for $72 \mathrm{~h}$ using an orbital shaker ( $150 \mathrm{rpm}$; Grant Instruments) housed inside the incubator ${ }^{17,18}$. At least three different biologically independent cell batches were studied for each experiment.

\section{Cell lysis, fractionation and western blotting}

Cells were lysed in ice-cold RIPA buffer (1\% Triton X$100,1 \%$ sodium deoxycholate, $2.5 \mathrm{mmol} / \mathrm{l}$ ethylenediaminetetraacetic acid (EDTA), $100 \mathrm{mmol} / \mathrm{l} \mathrm{NaCl}, 20 \mathrm{mmol} /$ 1 Tris-base; $\mathrm{pH}$ 7.4) supplemented with protease and phosphatase inhibitor cocktails (Roche). Lysates were incubated on ice for $45 \mathrm{~min}$ and then centrifuged for $10 \mathrm{~min}$ at $16,000 \times g$ to separate soluble from insoluble fractions.

Cell surface and cytosolic extracts were separated from nuclear. Cells were scraped, washed with phosphatebuffered saline ( $\mathrm{pH}$ 7.4), resuspended in hypotonic buffer (10 mM Hepes (pH 7.9), $1.5 \mathrm{mM} \mathrm{MgCl} 2,10 \mathrm{mM} \mathrm{KCl}$, 
$0.2 \mathrm{mM}$ phenylmethylsulfonyl fluoride and $0.5 \mathrm{mM}$ dithiothreitol) and allowed to swell on ice for $10 \mathrm{~min}$. After that 1\% NP40 was added and the cells were homogenised with a syringe and needle and vortexed for $2 \mathrm{~min}$. The nuclei were separated by spinning at $3300 \mathrm{~g}$ for $5 \mathrm{~min}$ at $4{ }^{\circ} \mathrm{C}$. The supernatant was used as soluble cytoplasmic/membrane extract. The nuclear pellet was extracted in nuclear extraction buffer $(20 \mathrm{mM}$ Hepes $(\mathrm{pH}$ 7.9), $100 \mathrm{mM} \mathrm{NaCl}, 1.5 \mathrm{mM} \mathrm{MgCl} 2,1 \%$ Triton, $1 \mathrm{mM}$ EDTA, $1 \mathrm{mM}$ EGTA, $10 \%$ glycerol, $0.5 \%$ deoxycholate, $0.1 \%$ SDS with protease and phosphatase inhibitor cocktails (Roche)) for $30 \mathrm{~min}$ on ice and centrifuged at $12,000 \times g$ for $30 \mathrm{~min}$. The supernatant was used as a nuclear extract.

Soluble nuclear and cytoplasmic/membrane protein lysates were analysed by SDS-PAGE and immunoblotting. Bound antibodies were visualised with horseradish peroxidase-conjugated anti-IgG antibodies and enhanced chemiluminescence susbtrates (Thermo Scientific). Antibodies for western blotting were obtained from the following sources: anti-cleaved caspase-3, total caspase-3, $\beta$-catenin, eNOS, eNOS phosphoS1177, Calnexin, cIAP1, TBP (Cell Signalling), $\beta$-catenin, active $\beta$-catenin, VEcadherin, eNOS phosphoS635, eNOS phosphoS114 (BD Biosciences), NOS3, PDHX and GAPDH (Santa Cruz).

\section{cGMP ELISA}

Lysates were extracted by addition of $0.1 \mathrm{~mol} / \mathrm{L} \mathrm{HCl}$ supplemented with $1 \mathrm{mmol} / \mathrm{L}$ 3-isobutyl-1-methylxanthine. cGMP concentration was assessed following acetylation using Cyclic GMP EIA Kit (Cayman Chemicals) according to the manufacturer's instructions. Resulting cGMP concentrations were normalised to protein content per sample.

\section{Immunostaining and confocal microscopy}

HUVEC on fibronectin-coated glass plates (In Vitro Scientific IBL) were fixed with $4 \%$ paraformaldehyde solution for $20 \mathrm{~min}$, permeabilized with $0.1 \%$ Triton X100 in PBS for 5 min and blocked with 5\% BSA in PBS for a further $30 \mathrm{~min}$. Cells were incubated overnight at $4{ }^{\circ} \mathrm{UF}$ $\mathrm{C}$ with anti-cleaved caspase- 3 antibody (cell signalling), active $\beta$-catenin (BD biosciences) or VE-Cadherin (BD Biosciences) or active $\beta$-catenin (Millipore) and/or 4',6diamidino-2-phenylindole (DAPI) to stain nuclei. Images were generated with a Nikon Spinning disk confocal microscope using a $\times 20$ objective and Nikon software. The percentage of cleaved caspase- 3 positive cells was calculated in at least 16 randomly selected fields of view for each condition, covering $\sim 2000-4000$ cells to estimate the level of apoptosis.

\section{En face staining of mouse aortas}

All procedures were conducted in accordance with the Directive 2010/63/EU of the European Parliament on the protection of animals used for scientific purposes, as enforced by national legislation, the UK Animal (Scientific Procedures) Act 1986 (as amended), under authorisation of the UK Home Office (Project License No. 70-8934). Male, 8-week-old C57BL/6J mice were purchased from Charles River Laboratories (Harlow, UK), maintained on a 12-hour day/night cycle, and fed a standard breeding/ maintenance chow ad libitum (RM3, Special Diets Services, UK) for 2 weeks prior to tissue harvest. Mice were terminally anaesthetised with an overdose of pentobarbitone $(120 \mathrm{mg} / \mathrm{kg}$ i.p.) and then perfused transcardially with an ice-cold $0.9 \%$ saline, $100 \mathrm{U} / \mathrm{ml}$ heparin solution, followed by ice-cold $4 \%$ paraformaldehyde (Parafix, Pioneer Research Chemicals Ltd, UK). Aortas were removed intact from the heart to the renal bifurcation, further postfixed in $4 \%$ paraformaldehyde at $4{ }^{\circ} \mathrm{C}$ overnight, then micro-dissected under a stereomicroscope in $0.1 \mathrm{M}$ phosphate-buffered saline to produce en face preparations. Co-localisation of eNOS (C-20; Santa Cruz) and $\beta$-catenin (BD biosciences) was visualised in $\mathrm{EC}$ from wild-type C57BL/6 mice by en face staining of susceptible (inner curvature) or protected (outer curvature) regions of the aorta followed by laser scanning confocal microscopy. Nuclei were stained with DAPI.

\section{Proximity ligation assay (PLA)}

PLAs were carried out using a Duolink In Situ Detection Kit as previously described ${ }^{15}$. HUVEC were cultured in six-well plates until confluent and subjected to flow for $72 \mathrm{~h}$ using an orbital shaker. Following flow exposure, cells were fixed with $4 \%$ paraformaldehyde then permeabilized with $0.5 \%$ Triton ${ }^{\circledR} \mathrm{X}-100$. In situ PLA was carried out using rabbit anti-eNOS and mouse anti- $\beta$-catenin primary antibodies in combination with Duolink In Situ Red Detection Kit (Sigma). PLA was carried out according to the manufacturer's instructions. Afterwards cells were washed to eliminate excess of reagents and cells were counterstained with DAPI and VE-Cadherin (BD Biosciences) to visualise cell junctions and nuclei, respectively. Images were generated with a Nikon Spinning disk confocal microscope using a $\times 20$ objective and Nikon software NIS elements. PLA analysis was carried out on confocal images. Approximately 2000 cells from four independent experiments were quantified. The PLA signal intensity per cell was analysed using Image and the particle analysis function on PLA images and cells numbers calculated using the same function on DAPI images. A mask for the cell edge was generated with the VEcadherin image and imposed over the PLA image to measure signal inside and outside of the mask.

\section{Transferase dUTP nick end labelling (TUNEL) assay}

To detect DNA fragmentation in apoptotic cells, terminal TUNEL was performed as previously 
described $^{19}$. A Click-iT TUNEL Alexa Fluor 594 Imaging Assay was used following the manufacturer instructions (Life Technologies). Briefly cells were fixed using $4 \%$ paraformaldehyde in PBS for $15 \mathrm{~min}$ and followed by a permeabilization step with $0.25 \%$ Triton ${ }^{\circledR} \mathrm{X}-100$ for $20 \mathrm{~min}$. Cells were then treated with terminal deoxynucleotidyl transferase for $60 \mathrm{~min}$ at $37^{\circ} \mathrm{C}$ to allow the incorporation of modified dUTPs at the $3^{\prime}-\mathrm{OH}$ ends of fragmented DNA, followed by a Click-iT ${ }^{\circledR}$ reaction for $30 \mathrm{~min}$ at $37^{\circ} \mathrm{C}$ to label ends with a fluorescent dye through click chemistry. Afterwards cells were washed gently to eliminate excess of reagents and cells were counterstained with DAPI and VE-Cadherin (BD Biosciences) to visualise the cell edges and nuclei, respectively. Images were generated with a Nikon Spinning disk confocal microscope using a $\times 20$ objective and Nikon software NIS elements. The percentage of TUNEL positive cells was calculated in 16 selected fields of view for each condition, covering 2000-4000 cells to estimate the level of apoptosis.

\section{RNA isolation and quantitative RT-PCR}

RNA was isolated from cells using RNeasy Mini kits (Qiagen). Contaminating DNA was removed by oncolumn DNase digestion (Qiagen). cDNA was prepared using a High-Capacity Reverse Transcription Kit (Thermo Scientific). Quantitative real-time PCR (qPCR) was carried out with cDNA using SYBR green mastermix (Primer Design). GADPH was used as a reference gene. The qPCR oligonucleotide primers used for eNOS were: F: CATC TTCAGCCCCAAACGGA R: AGCGGATTGTAGCCTG GAAC; for Survivin: F: TGAGAACGAGCCAGACTTGG R: TGTTCCTCTATGGGGTCGTCA; for KLF2: F: TGG GCATTTTTGGGCTACCT R: CCCAGTTCCAAGCAA CCAGA; for E-SEL: F: GCTCTGCAGCTCGGACAT R: GAAAGTCCAGCTACCAAGGGAAT; for Bcl-2: F: AT GTGTGTGGAGAGCGTCAA R: GGGCCGTACAGTTC CACAAA; for GAPDH: F: CTATAAATTGAGCCCGCA GCC R: ACCAAATCCGTTGACTCCGA; for XIAP: F: AGTGTCTGGTAAGAACTACTG R: CCCATTCGTAT AGCTTCTTG; for WISP-1: F: TCATTAAGGCAGGG AAGAAG R: GTCTTAGACTTGTAGGGGATG; for BIRC3: F: ACAAGCAAGAGAACTGATTG R: GATCTG AAACATCTTCTGTGG and for Caspase-3: F: AAAGC ACTGGAATGACATC R: CGCATCAATTCCACAATT TC. The amplification process included one cycle of $10 \mathrm{~min}$ at $95^{\circ} \mathrm{C}, 40$ cycles for $15 \mathrm{~s}$ at $95^{\circ} \mathrm{C}$, followed by 40 cycles for $1 \mathrm{~min}$ at $60^{\circ} \mathrm{C}$. Thermal cycling and fluorescence detection were performed using an ABI 7500 Fast Prism (PE Applied Biosystems, Foster City, CA, USA).

\section{Apoptosis gene expression array}

HUVEC were cultured in six-well plates until confluent and subjected to flow for $72 \mathrm{~h}$ using an orbital shaker.
Following flow exposure, cells were washed twice with cold PBS and total DNA free mRNA was isolated using RNeasy Mini kits (Qiagen) and on-column DNase digestion as described above. mRNA integrity and concentration were determined spectrophotometrically. An RT2 Profiler $^{\mathrm{tm}}$ polymerase chain reaction (PCR) apoptosis array (PAHS-012ZA-Qiagen) was performed according to the manufacturer's instructions. Briefly, a total of $0.5 \mu \mathrm{g}$ of RNA per sample was used with the RT2 First Strand kit (Qiagen) to obtain cDNA after incubation for $5 \mathrm{~min}$ with the gDNA elimination buffer. The PCR amplification process included one cycle of $10 \mathrm{~min}$ at $95^{\circ} \mathrm{C}, 40$ cycles for $15 \mathrm{~s}$ at $95^{\circ} \mathrm{C}$, followed by 40 cycles for $1 \mathrm{~min}$ at $60^{\circ} \mathrm{C}$. Thermal cycling and fluorescence detection were performed using an ABI 7500 Fast Prism (PE Applied Biosystems, Foster City, CA, USA). The signals of the target cDNAs were normalised by comparison with the housekeeping genes HPRT1 supplied within the 96-well microtiter plate. The normalised amount of each target mRNA present in each condition was calculated using a comparative Ct method and using a web-based PCR array data analysis tool (https://www.qiagen.com/gb/shop/ genes-and-pathways/data-analysis-center-overview-page/) with 1.5-fold difference set as baseline.

\section{Statistical analysis}

All data are presented as mean \pm SEM. Statistical analysis was performed using GraphPad Prism software (v7.1). Statistical significance was assessed using paired two-tailed $t$-test (for comparing two conditions) or by one-way analysis of variance (ANOVA) with repeated measures for multiple conditions. Each $n$ is generated with a separate batch of ECs. For HUVEC, these are pools of cells from different donors and for HAEC, in each batch EC had been isolated from a single donor. When using one-way ANOVA, tests for equal variance were run and if significantly different standard deviations were found (thus different variances) in the ANOVA with repeated measures a Gaussian distribution and no sphericity (not equal variability of the differences) were assumed and the Greenhouse-Geisser correction was applied.

\section{Results \\ $\beta$-catenin regulates eNOS activity in static HUVEC}

To investigate whether $\beta$-catenin regulates eNOS activation in HUVEC, we assessed eNOS phosphorylation in cells following transfection with a pool of $\beta$-catenin siRNA oligonucleotides or an siRNA scrambled control pool. $\beta$-catenin depletion did not alter basal levels of eNOS phosphorylation (Figs. 1a, b and S1a, b), however, it did reduce agonist-induced eNOS phosphorylation on Ser1177 (Figs. 1a and S1a). Interestingly, $\beta$-catenin depletion had no effect on agonist-induced eNOS phosphorylation on Ser633 

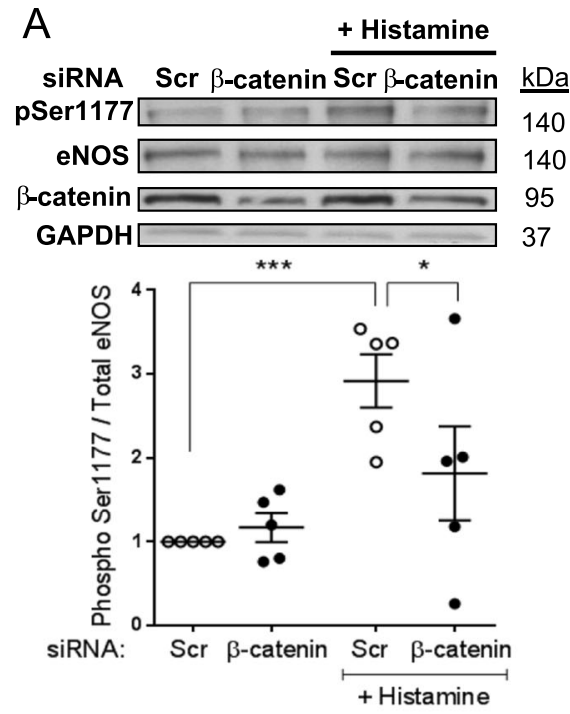

B

+ Histamine

siRNA Scr $\beta$-catenin Scr $\beta$-catenin $\mathrm{kDa}$ pSer633 — — 140

eNOS 140

$\beta$-catenin $\square 95$

GAPDH 37

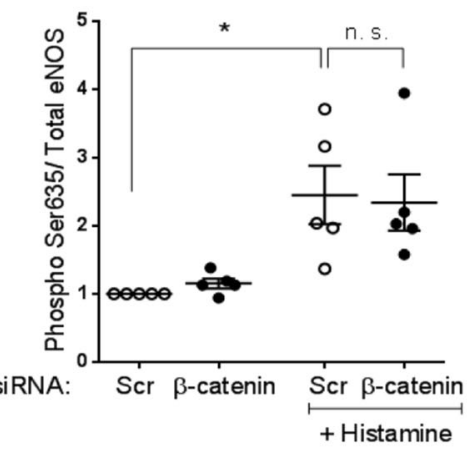

C

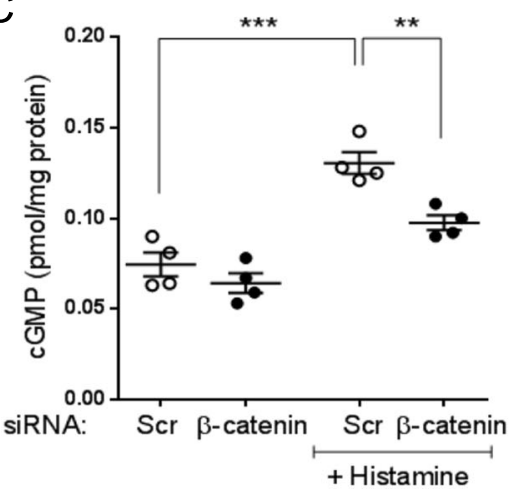

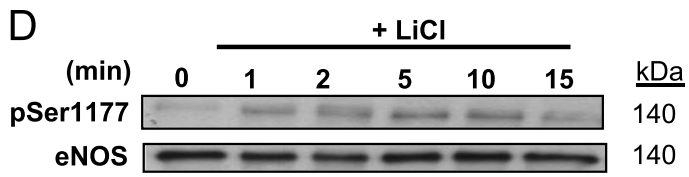

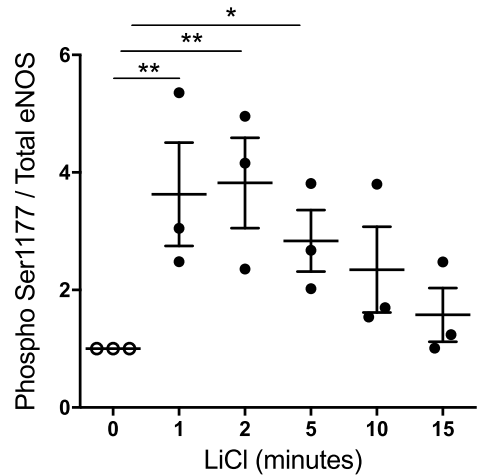

$E$
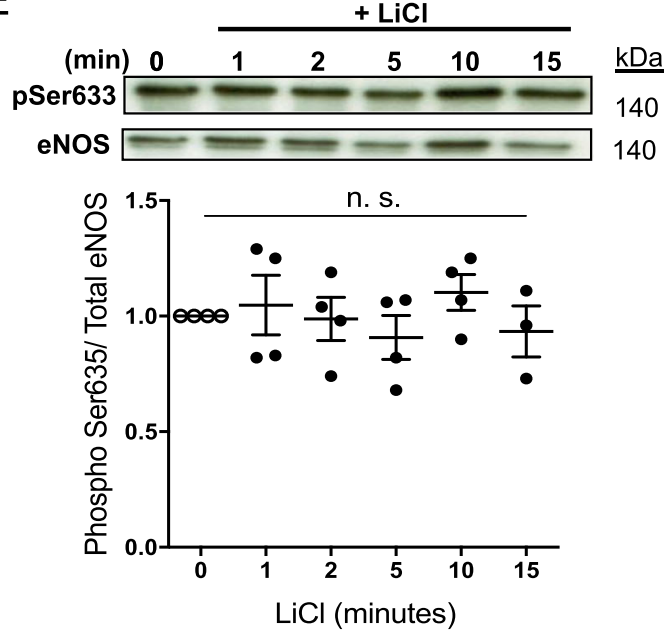

F
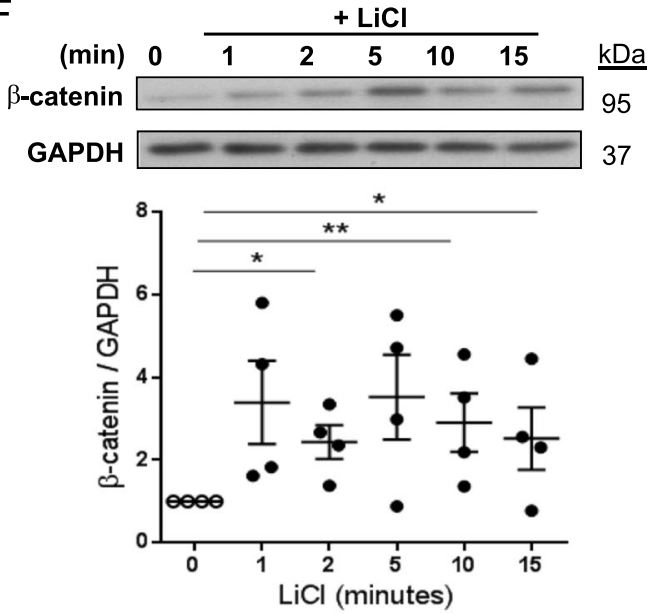

Fig. 1 (See legend on next page.) 
(see figure on previous page)

Fig. $1 \beta$-catenin depletion inhibits agonist-induced eNOS phosphorylation in static HUVEC. a-c HUVEC were transfected with siRNA targeting $\beta$-catenin (100 nM) or scrambled control siRNA (Scr) and cultured for $72 \mathrm{~h}$ before treatment for $5 \mathrm{~min}$ with vehicle or histamine $(100 \mathrm{mmol} / \mathrm{L})$. Cell lysates were analysed by western blot using total eNOS, b-catenin, phospho-Ser1177 (a) or phospho-Ser633 (b) antibodies. c cGMP levels were quantified by ELISA and results expressed relative to protein content per sample (shown relative to scrambled control; $n=4$ ). $\mathbf{d}-\mathbf{f}$ HUVEC were cultured for $72 \mathrm{~h}$ before treatment for the indicated times with vehicle or $\mathrm{LiCl}(20 \mathrm{mM})$. Cell lysates were analysed by western blot using total eNOS, phospho-Ser1177 (d) or phospho-Ser633 (e) and b-catenin (f) antibodies. a, b, d, e Results expressed as the densitometric ratio of phospho-eNOS/ GAPDH to total eNOS/GAPDH and shown relative to untreated control $(n=5)$; analysis by one-way ANOVA with repeated measures, ns nonsignificant, ${ }^{*} p \leq 0.05,{ }^{* *} p \leq 0.01,{ }^{* * *} p \leq 0.001$.

(Figs. 1b and S1b). Intracellular cGMP levels are a wellestablished indicator of bioactive NO levels; we observed that in HUVEC, the histamine-induced increase in cGMP production was reduced following depletion of $\beta$-catenin with siRNA (Fig. 1c). The absence of $\beta$-catenin protein in HUVEC transfected with $\beta$-catenin siRNA was confirmed by western blotting (Fig. S1c), furthermore, eNOS expression was not altered in $\beta$-catenin knockdown static cells (Fig. S1d).

To confirm that $\beta$-catenin can promote eNOS phosphorylation on Ser1177 and thus increase NO and cGMP production, we treated HUVEC with $\mathrm{LiCl}$ that inhibits GSK3 $\beta$-dependent phosphorylation of $\beta$-catenin and promotes its accumulation. We found that elevating $\beta$-catenin levels with $\mathrm{LiCl}$ increased eNOS phosphorylation on Ser1177 but had no effect on Ser633 in static HUVEC (Fig. 1d-f). Together these data demonstrate that $\beta$-catenin enhances agonist-dependent eNOS activation and subsequent cGMP production in HUVEC under static conditions.

\section{$\beta$-catenin mediates the anti-apoptotic effects of NO-cGMP in static HUVEC}

Since reduction of $\beta$-catenin may impair eNOS function and since $\mathrm{NO}$ is an important factor for $\mathrm{EC}$ survival $^{7}$, we assessed the potential protective effects of $\beta$-catenin, in relation to NO signalling, in static cultures following induction of apoptosis. In wild-type MPEC, application of an NO donor (S-nitroso-N-acetylpenicillamine; SNAP) attenuated TNF $\alpha$-induced apoptosis, indicated by a lower number of cleaved caspase- 3 positive cells in the presence of SNAP, however, in $\beta$-catenin ${ }^{-1-}$ MPEC, the protective effects of SNAP on TNF $\alpha$-induced apoptosis were abrogated (Fig. 2a). We also examined TNFa-induced apoptosis in HUVEC and observed that siRNA depletion of $\beta$-catenin reduced the anti-apoptotic action of SNAP in this context (Figs. 2b, e and S1e), demonstrating that $\beta$-catenin also mediates the pro-survival effects of NO in human EC under static conditions. Since $\beta$-catenin appears to regulate the level of activated eNOS and subsequent cGMP production in HUVEC, we examined activation of caspase- 3 by $\mathrm{H}_{2} \mathrm{O}_{2}$ in HUVEC in the presence of sildenafil, a pharmacological sGC activator.
Whilst cleaved caspase- 3 levels induced by $\mathrm{H}_{2} \mathrm{O}_{2}$ are reduced by sildenafil treatment in scrambled controltreated cells, this was not the case in $\beta$-catenin depleted HUVEC (Fig. 2c). Furthermore, ODQ, a pharmacological sGC inhibitor, abrogated the protective effects of SNAP in the presence of $\mathrm{H}_{2} \mathrm{O}_{2}$ (Fig. 2d), indicating that $\mathrm{NO}$ exerts its anti-apoptotic effects through sGC activation and cGMP production. Together these data suggest that $\beta$-catenin mediates the pro-survival effects of $\mathrm{NO}$ and cGMP upon pharmacological (non-physiological) induction of apoptosis in static EC. eNOS expression and activation are known to increase in EC exposed to $\mathrm{UF}^{18}$ and enhanced endothelial NO synthesis promotes survival of EC under physiological levels of shear stress against TNF $\alpha$ and $\mathrm{H}_{2} \mathrm{O}_{2}{ }^{8,9}$. For these reasons, we next sought to determine whether $\beta$-catenin mediates the pro-survival effects of NO under physiological flow conditions.

\section{$\beta$-catenin associates with eNOS in HUVEC exposed to physiological flow}

To confirm our previous observation made in static $\mathrm{EC}^{15}$, and to test whether eNOS and $\beta$-catenin interact under physiological flow conditions, we carried out a PLA in HUVEC subjected to flow for $72 \mathrm{~h}$. We used an orbital shaking platform to generate reproducible spatially separated atheroprotective (undisturbed; uniaxial, high wall shear stress) and atheroprone (disturbed; multiaxial, low wall shear stress) flow patterns ${ }^{17,18}$. As previously described, HUVEC growing under atheroprotective, UF were elongated and aligned meanwhile the cells in the atheroprone, DF central region showed a non-aligned mosaic-like morphology (Figs. 3a and S2a). Furthermore, $\mathrm{EC}$ in the UF region expressed high levels of KLF2 and KLF4 and lower levels of MCP-1 and E-selectin compared to EC exposed to $\mathrm{DF}^{20,21}$ (Fig. S2b). Co-localisation was observed in EC fixed and stained with anti-eNOS and anti- $\beta$-catenin antibodies (Fig. S2a) and amplification products were detected by PLA indicating proximity $(<40 \mathrm{~nm})$ of both proteins in HUVEC exposed to both UF and DF (Fig. 3a) but not in controls stained separately with either anti-eNOS or anti- $\beta$-catenin antibody. The average PLA signal per cell was lower in EC exposed to UF (Fig. 3a) consistent with increased eNOS activity ${ }^{15}$. 


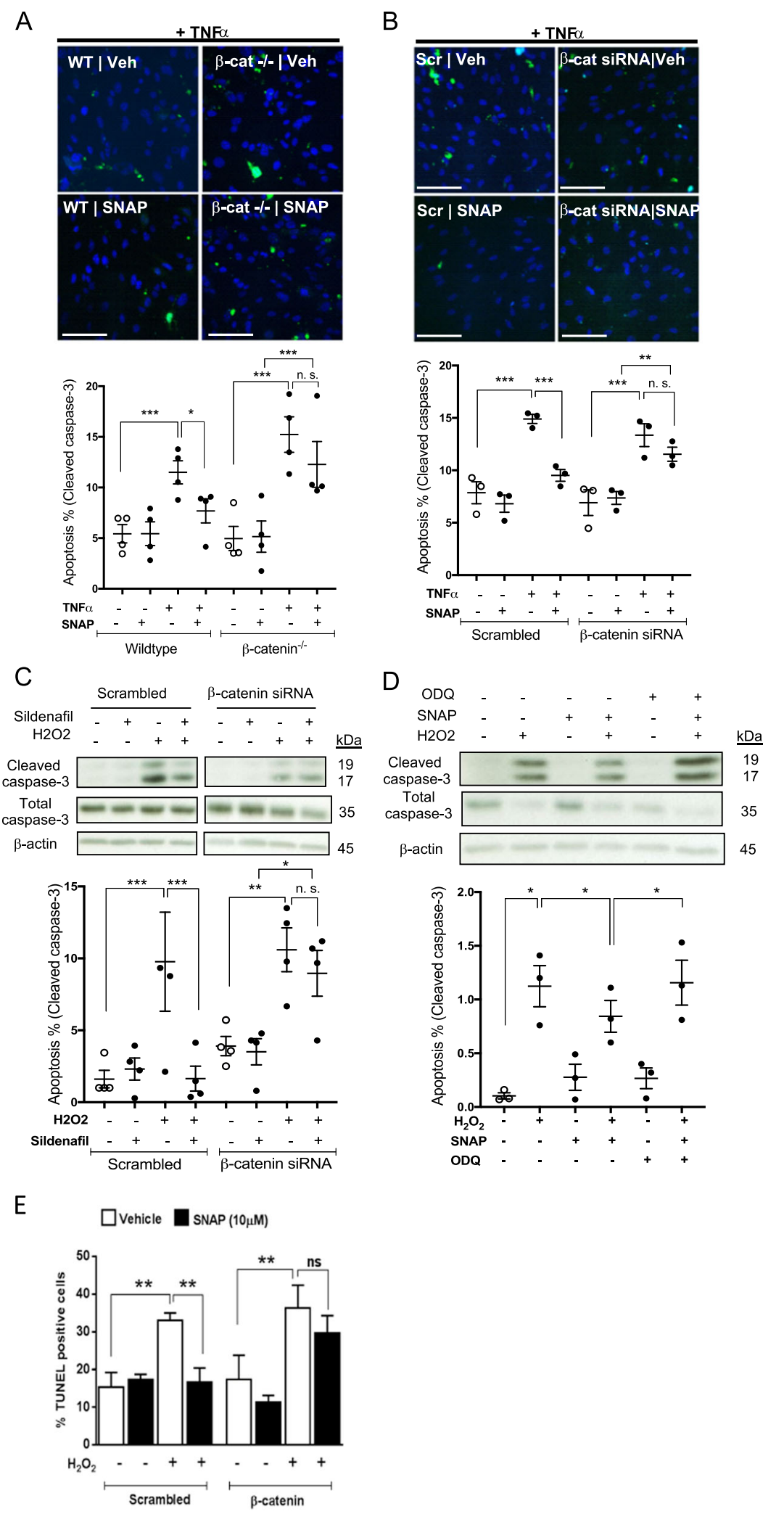

Fig. 2 (See legend on next page.) 
(see figure on previous page)

Fig. $2 \beta$-catenin mediates the anti-apoptotic effects of NO in static HUVEC. a Wild-type and $\beta$-catenin ${ }^{-1-}$ MPECS were treated with SNAP $(10 \mu \mathrm{M})$ or vehicle. $\mathbf{b}$ HUVEC were transfected with siRNA targeting $\beta$-catenin $(100 \mathrm{nM})$ or scrambled control and cultured for $72 \mathrm{~h}$ before treatment with TNFa $(10 \mathrm{ng} / \mathrm{ml})$ for $18 \mathrm{~h}$ in the presence or absence of SNAP $(10 \mu \mathrm{M})$. $\mathbf{a}, \mathbf{b}$ EC were fixed and incubated with anti-cleaved caspase-3 antibody and DAPI. Representative images are shown. The percentage of cleaved caspase-3 positive cells was calculated in five randomly selected fields of view $(n=4)$. c HUVEC were transfected with siRNA targeting $\beta$-catenin $(100 \mathrm{nM})$ or scrambled control and cultured for $72 \mathrm{~h}$ before treatment with $\mathrm{H}_{2} \mathrm{O}_{2}(200 \mu \mathrm{M})$ for $6 \mathrm{~h}$ in the presence or absence of sildenafil $(50 \mathrm{nM} ; n=4)$. The bands shown by way of example are from the same experiment. d HUVEC were treated with $\mathrm{H}_{2} \mathrm{O}_{2}(200 \mu \mathrm{M})$ for $6 \mathrm{~h}$ in the presence of SNAP $(10 \mu \mathrm{M})$, ODQ $(10 \mu \mathrm{M})$ or both in combination $(n=3)$. $\mathbf{c}$, d Cleaved $(17$ and $19 \mathrm{kDa}$ ) and full length $(35 \mathrm{kDa}$ ) caspase-3 were detected in western blots of cell lysates and normalised to $\beta$-actin. Densitometry values are shown expressed relative to vehicle control. e HUVEC were transfected with siRNA targeting $\beta$-catenin $(100 \mathrm{nM})$ or scrambled control and cultured for $72 \mathrm{~h}$ before treatment with $\mathrm{H}_{2} \mathrm{O}_{2}(200 \mu \mathrm{M})$ for $6 \mathrm{~h}$ in the presence or absence of SNAP $(10 \mu \mathrm{M}$, following which cells were fixed and DNA fragmentation assessed using a Click-IT TUNEL Imaging Kit $(n=5)$. All analyses by one-way ANOVA with repeated measures, ns non-significant, ${ }^{*} p \leq 0.05,{ }^{* *} p \leq 0.01$, ${ }^{* * *} p \leq 0.001$. Scale bars show $100 \mathrm{~mm}$.
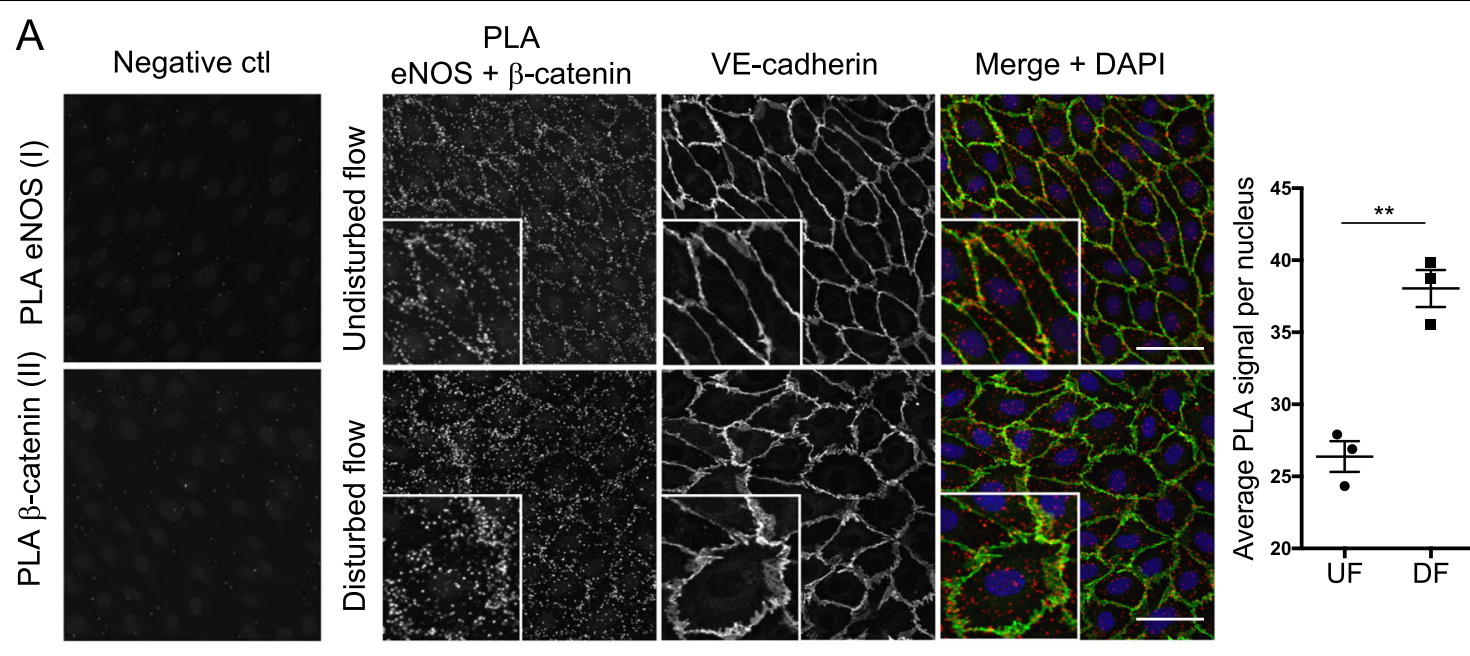

\section{B}

$\beta$-catenin

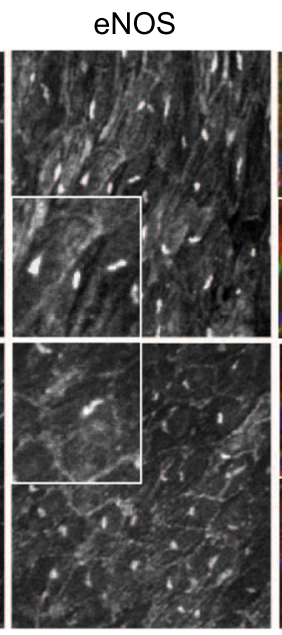

Merge + DAPI
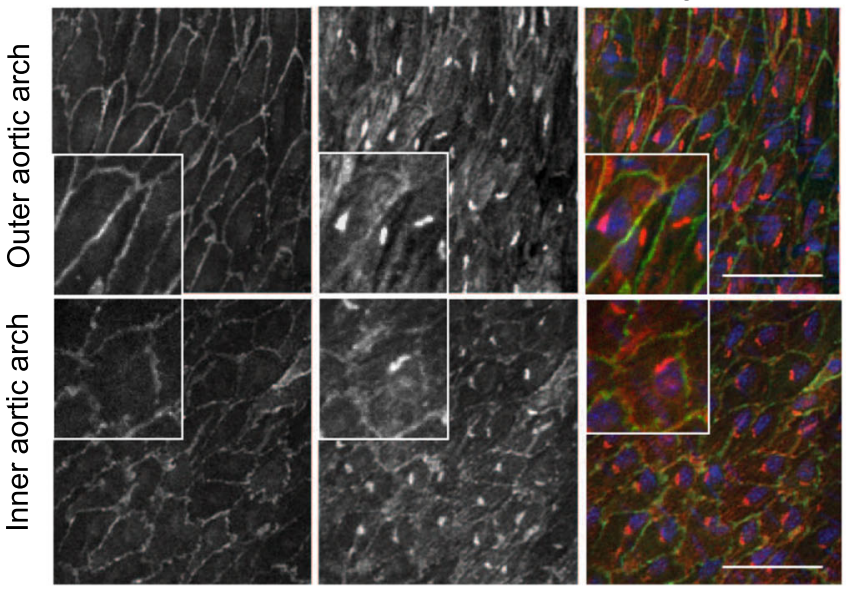

Fig. 3 -catenin interacts with eNOS under flow. a HUVEC were exposed to orbital flow for $72 \mathrm{~h}$, fixed and subject to PLA using antibodies targeting eNOS and b-catenin. In control samples, PLA was carried out in the absence of b-catenin (I) or eNOS antibody (II). VE-cadherin and DAPI were used to stain cell junctions and nuclei, respectively. Representative images are shown and the average PLA signal quantified in HUVEC under disturbed flow (DF) or undisturbed flow (UF; $n=4$ ); analysis by paired Student's $t$ test, ${ }^{* *} p \leq 0.01$. b EC from protected (outer curvature) or atherosusceptible (inner curvature) regions of the mouse aorta were stained with eNOS (red) and $\beta$-catenin (green) antibodies and imaged en face by confocal microscopy. Merged representative images with DAPI (blue) are shown $(n=3)$. Scale bars show $50 \mathrm{~mm}$. 
$\beta$-catenin and eNOS also co-localise in vivo in ECs of the inner and outer curvature of the aortic arch, regions of disturbed and UF, respectively, however, co-localisation was more prominent in the inner aortic arch (Fig. 3b). In contrast with observations made in HUVEC subjected to acute low magnitude shear stress ${ }^{22}$, no difference in the subcellular localisation of $\beta$-catenin was observed in HUVEC exposed to chronic flow using an orbital shaker (Fig. S2c).

\section{$\beta$-catenin regulates eNOS phosphorylation in HUVEC exposed to physiological flow}

Since activation of eNOS causes dissociation from $\beta$-catenin in static cells ${ }^{15}$, the higher level of interaction observed in EC exposed to DF suggests lower activation of eNOS in these cells. We thus measured eNOS phosphorylation on Ser1177, Ser633 and Ser114 and observed lower eNOS phosphorylation in EC exposed to DF compared to those exposed to UF (Fig. 4a). To investigate whether $\beta$-catenin regulates eNOS activation in HUVEC exposed to flow, we assessed eNOS phosphorylation in HUVEC following transfection with $\beta$-catenin siRNA or a siRNA scrambled control and exposed to flow for $72 \mathrm{~h}$ (Fig. 4b). We observed that eNOS phosphorylation on Ser1177 decreased in EC exposed to UF when $\beta$-catenin was depleted but not in EC exposed to DF, consistent with our findings in static EC. eNOS expression was not altered by $\beta$-catenin knockdown, compared to scrambled control transfection, although eNOS expression was different in EC exposed to DF compared to UF (Fig. S2d); but interestingly, phosphorylation on Ser114 was reduced in both UF- and DF-exposed HUVEC when $\beta$-catenin was depleted (Fig. 4b). In contrast, treatment of HUVEC with $\mathrm{LiCl}$ increased Ser1177 phosphorylation in both regions (Fig. 4c), confirming that $\beta$-catenin interacts with and can activate eNOS under physiological and atheroprone flow conditions.

\section{Deletion of $\beta$-catenin increases apoptosis only in HUVEC exposed to disturbed flow}

Atheroprotective, laminar flow activates eNOS and is the predominant source of NO in ECs. It has also been associated with EC survival ${ }^{8,10}$. In contrast, atherogenic shear stress profiles are associated with reduced expression of pro-survival genes and increased apoptosis, both in vitro and in vivo $^{23,24}$. We measured apoptosis by cleaved caspase-3 staining of HUVEC subjected to flow using an orbital shaker and observed that the percentage of apoptosis was higher under DF compared to UF as expected (Fig. S3a). Having established the involvement of $\beta$-catenin in NO-mediated anti-apoptotic activity in static cultures, we sought to determine the pro-survival actions of $\mathrm{NO}$ and $\beta$-catenin under physiological flow conditions. Inhibition of sGC activity with ODQ in HUVEC subjected to shear stress for $72 \mathrm{~h}$, increased apoptosis of HUVEC exposed to DF as assessed by cleaved caspase-3 immunostaining (Fig. 5a). Furthermore, depletion of $\beta$-catenin in HUVEC promoted apoptosis exclusively in HUVEC exposed to DF (Figs. 5b and S3b). In agreement with the higher level of apoptotic cells observed in DF-exposed HUVEC, we also detected higher levels of cleaved caspase-3 in lysates from HUVEC subjected to DF for $72 \mathrm{~h}$ (Fig. S3c). Unexpectedly, we found that the expression level of pro-caspase-3 $(35 \mathrm{kDa})$ was lower in lysates from EC exposed to UF compared to DF (Fig. 5c), though caspase- 3 mRNA expression showed only a $20 \%$ increase in cells exposed to DF compared to UF (Fig. S3d), suggesting that a post-translational mechanism may be regulating the protein expression and/or stability of caspase3 in HUVEC exposed to UF.

\section{Inhibition of $\beta$-catenin transcriptional activity increases apoptosis in HUVEC exposed to disturbed flow}

Since $\beta$-catenin can regulate the expression of prosurvival genes and has also been shown to act downstream of eNOS/NO/cGMP, we investigated whether inhibiting its transcriptional activity also influenced cell survival under flow conditions. Inhibition of $\beta$-catenindependent transcriptional activation with the specific inhibitors of $\beta$-catenin/TCF-LEF interaction, iCRT5 or $\mathrm{FH} 535^{25,26}$, also exclusively increased apoptosis in HUVEC under DF in the same conditions (Figs. $5 \mathrm{~d}$ and S3e) and in HAEC exposed to DF (Fig. S3f). The low level of apoptosis observed under UF was not altered by any method used to manipulate eNOS or $\beta$-catenin signalling (Figs. 5a-e and $\mathrm{S} 3 \mathrm{e}-\mathrm{g}$ ). Whilst treatment with histamine, which upregulates eNOS, reduced apoptosis in HUVEC exposed to DF (Fig. 5e), inhibition of eNOS with $100 \mathrm{mM}$ L-NAME did not affect apoptosis in HUVEC exposed to either DF or UF (Fig. S3g). These data indicate that signalling through sGC and $\beta$-catenin is essential to maintain cell survival in EC exposed to DF and that other additional mechanisms contribute to cell survival in UFexposed HUVEC.

\section{Inhibition of $\beta$-catenin-dependent transcription downregulates the expression of anti-apoptotic genes in HUVEC under flow}

As the inhibition of cGMP signalling and $\beta$-catenindependent transcription increases apoptosis in HUVEC exposed to DF, we studied the expression of several antiapoptotic genes that are potential targets of $\beta$-catenin: XIAP, WISP-1, Bcl-2 and survivin (BIRC5). Bcl-2 and survivin exhibited differential expression between DF and UF regions in HUVEC (Fig. 6a, b) as did eNOS (Fig. 5c). Interestingly, survivin mRNA expression was reduced when cells were treated with iCRT5 or FH535, in HUVEC exposed to both UF and DF (Figs. 6a and S4a). Bcl-2 and 


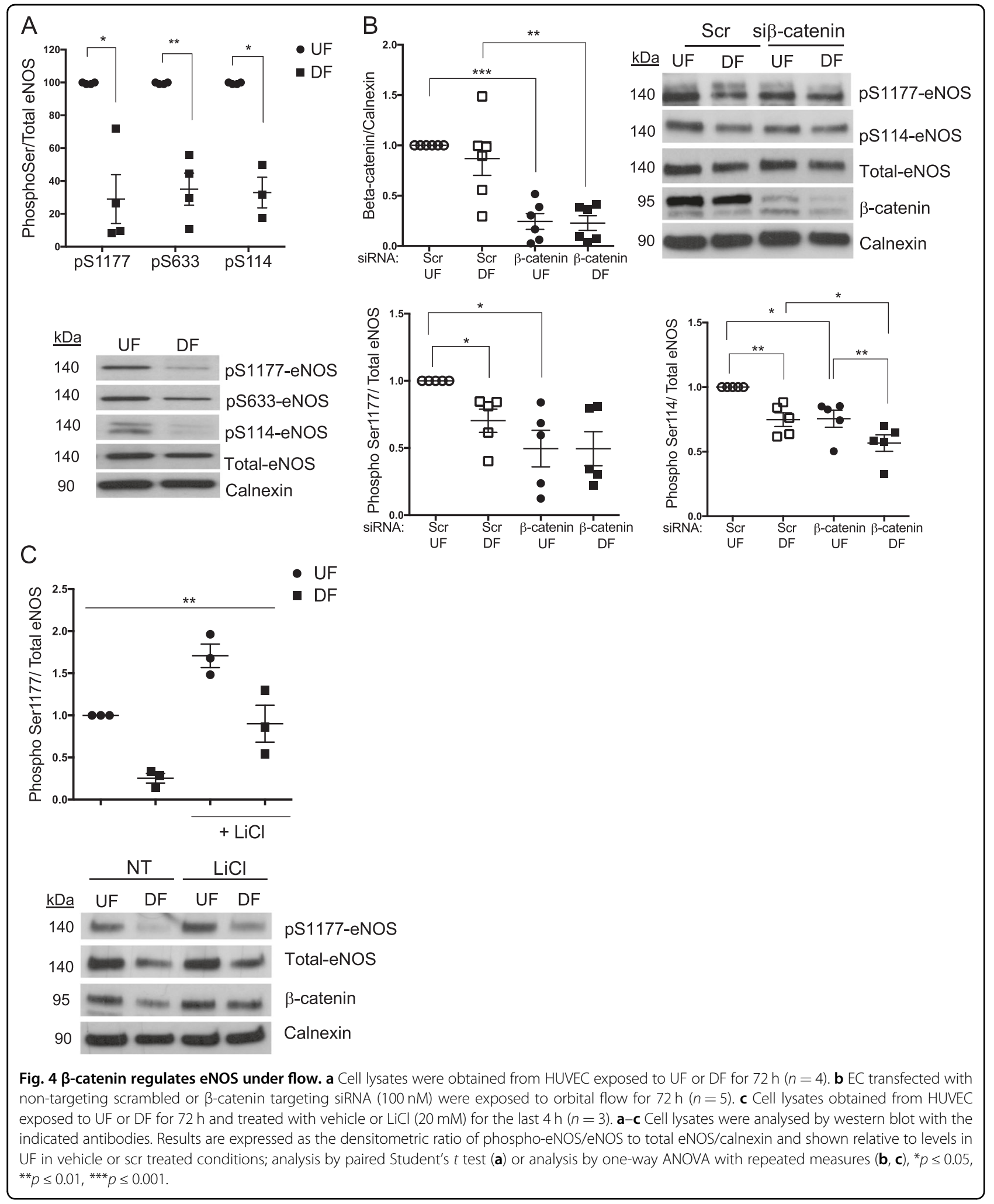

eNOS expression were also reduced in cells under UF in the presence of iCRT5. Treatment of HUVEC with ODQ also reduced the expression of eNOS (Fig. 6d), suggesting the presence of a positive feedback mechanism in the eNOS-sGC- $\beta$-catenin pathway. WISP-1 showed very low expression in HUVEC and no differences were found in 

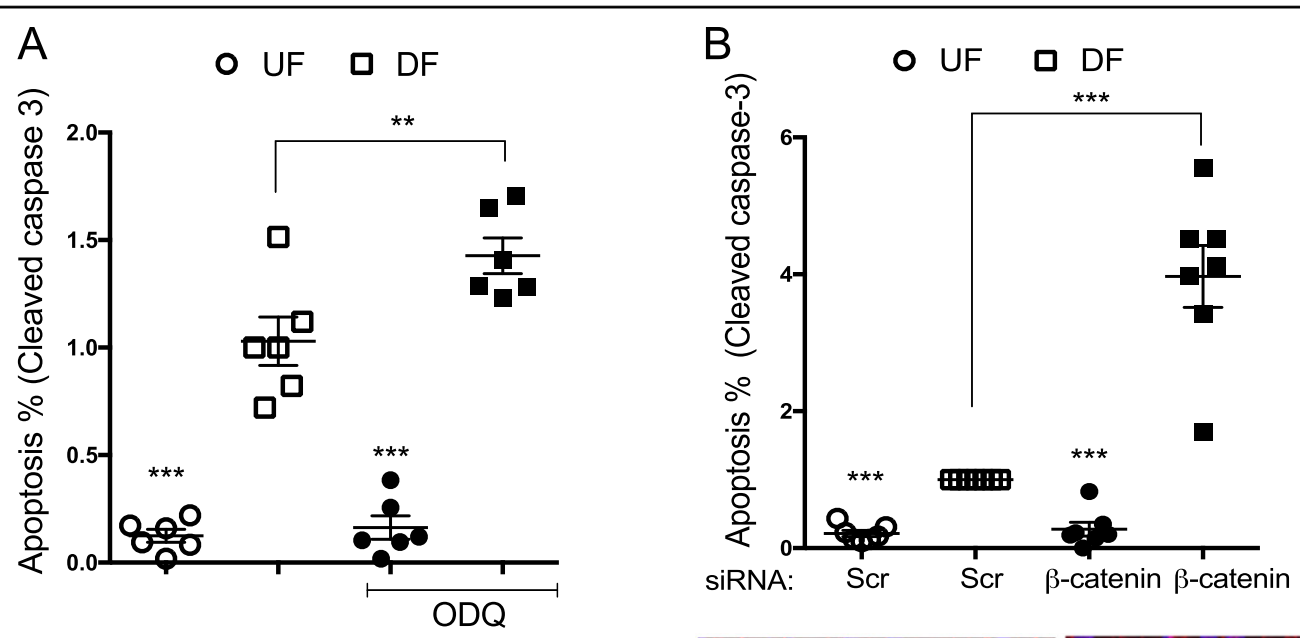

C
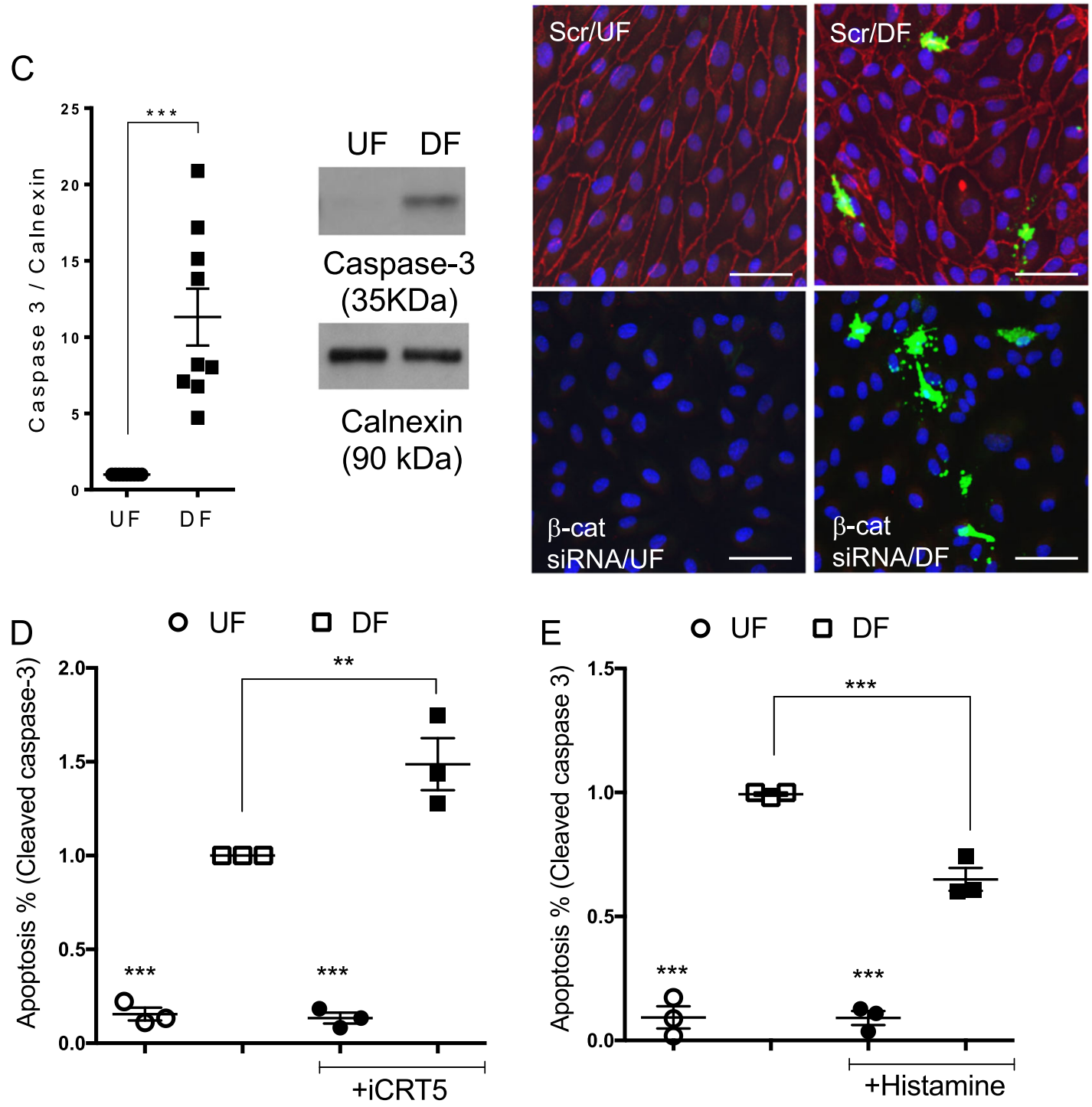

Fig. 5 (See legend on next page.) 
(see figure on previous page)

Fig. $\mathbf{5}$ Inhibition of sGC and $\beta$-catenin transcriptional activity increases apoptosis in HUVEC exposed to disturbed flow. a-e HUVEC were exposed to orbital flow for $72 \mathrm{~h}$ and treated with DMSO, ODQ $(10 \mathrm{mM})(\mathbf{a})$, iCRT5 $(50 \mathrm{mM})(\mathbf{d})$ or Histamine $(100 \mathrm{mmol} / \mathrm{L})(\mathbf{e})$ for the last $24 \mathrm{~h}$ of flow exposure or transfected with b-catenin or Scr siRNA (100 nM) (b). b ECs were fixed and incubated with a cleaved caspase-3 antibody (green), anti-bcatenin (red) and nuclei stained with DAPI. Representative images are shown. $\mathbf{a}, \mathbf{b}, \mathbf{d}$, e The percentage of cleaved caspase-3 positive cells was quantified in regions of undisturbed (UF) or disturbed flow (DF) ( $n=3-7)$. c HUVEC were exposed to orbital flow or static conditions for $72 \mathrm{~h}$. Full length ( $35 \mathrm{kDa}$ ) caspase-3 was detected in western blots of cell lysates. Results are expressed as the densitometric ratio of caspase-3 to calnexin. Representative western blots are shown (right panel) $(n=9)$; analysis by paired Student's $t$ test $\mathbf{c}$ or analysis by one-way ANOVA with repeated measures $(\mathbf{a}, \mathbf{b}, \mathbf{d}, \mathbf{e}),{ }^{*} p \leq 0.05,{ }^{* *} p \leq 0.01,{ }^{* * *} p \leq 0.001$. Scale bars show $50 \mathrm{~mm}$.
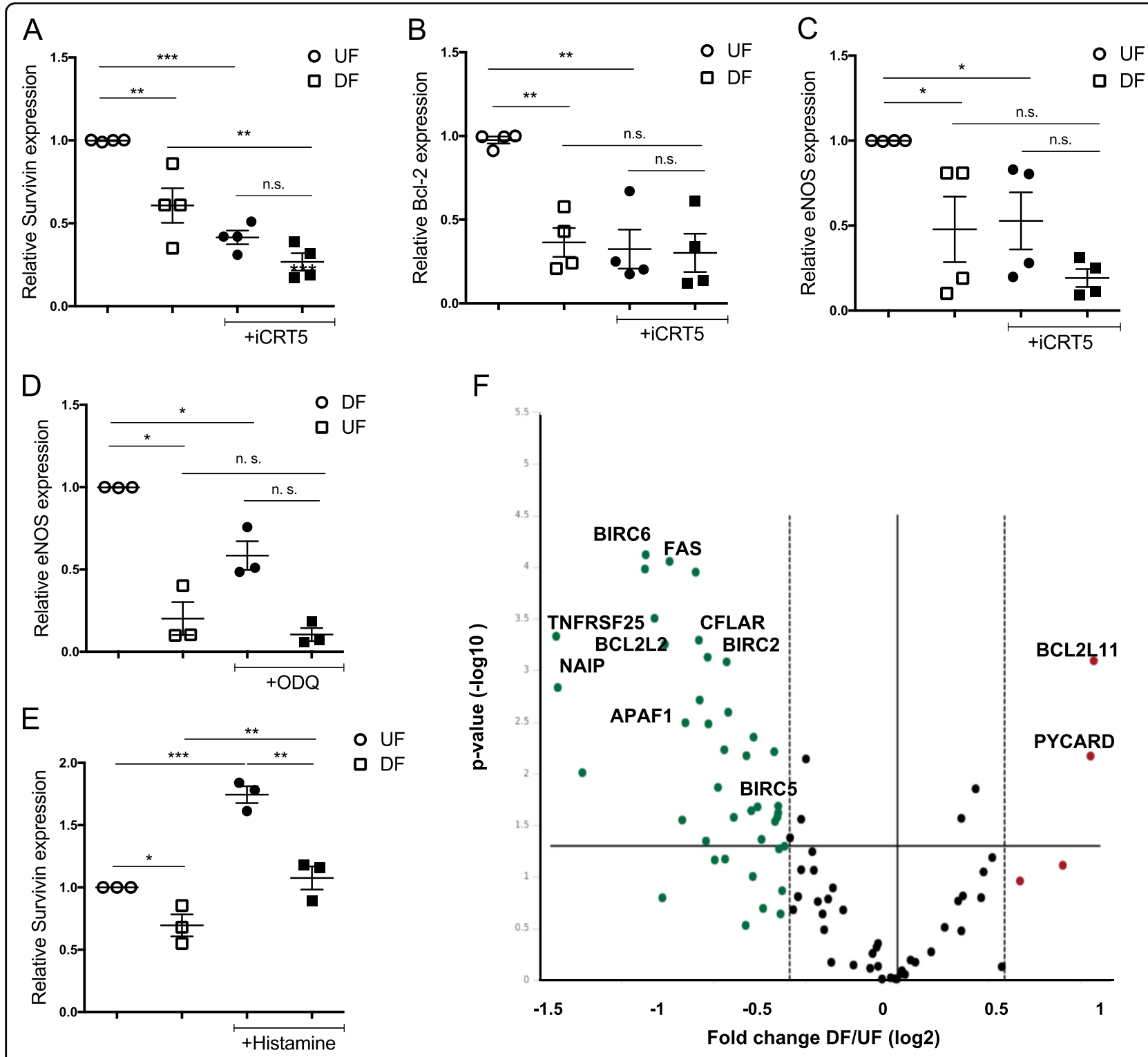

Fig. 6 Inhibition of $\beta$-catenin downregulates the expression of survivin and Bcl-2 in HUVEC exposed to flow. a-f HUVEC were exposed to orbital flow for $72 \mathrm{~h}$ and treated with DMSO or iCRT5 $(50 \mu \mathrm{M})(\mathbf{a}-\mathbf{c})$, ODQ $(10 \mathrm{mM})(\mathbf{d})$ or histamine $(100 \mathrm{mM})(\mathbf{e})$ for the last $24 \mathrm{~h}$ of flow or left untreated (f). Transcript levels of survivin (a, e), Bcl-2 (b) and eNOS (c, d) were assessed in HUVEC under disturbed flow (DF) or undisturbed flow (UF) by qRT-PCR using GAPDH as a housekeeping gene. Values are shown relative to expression in vehicle treated HUVEC under UF; analysis by ANOVA with repeated measures $(n=3-4)$; ns not significant, ${ }^{*} p \leq 0.05,{ }^{* *} p \leq 0.01,{ }^{* * *} p \leq 0.001$. f mRNA samples from HUVEC exposed to DF or UF were analysed using an mRNA expression array targeting 84 apoptotic-related genes. HPRT1 was used as housekeeping gene $(n=3)$. The Volcano plot shows expression fold change of mean across replicates in each condition from DF to UF on $x$-axis $(\log 2)$ and $p$ value $(-\log 10)$ on $y$-axis. Significantly differentially expressed genes $(p$ value $<0.05$ ) are highlighted in red (upregulated; FC > 1.5) or green (downregulated; $F C<-1.5$ ). Relevant genes are indicated. 
expression of XIAP between HUVEC exposed to UF and DF in the presence or absence of iCRT5 (not shown).

To identify novel putative genes regulating apoptosis and cell survival in EC exposed to flow, an apoptosis transcriptome array was performed with HUVEC exposed to UF or DF using an orbital shaker for $72 \mathrm{~h}$ (Table S1). We classified the genes upregulated and downregulated in DF conditions according to the apoptotic pathways they regulate $^{27}$ and their function as shown in Table 1 . We observed that the most significant change in EC exposed to DF was the downregulation of anti-apoptotic genes (Figs. $6 \mathrm{f}$ and S5). These include members of the BCL and IAP (inhibitors of apoptosis) families including BIRC5 (survivin), NIAP1, BIRC2 (cIAP1), BIRC6, BCL2L2 and MCL1. Although BIRC3 expression did not change from UF to DF, it was reduced in EC treated with iCRT5 (Fig. S4b) and constitutes a novel target of $\beta$-catenin in EC. BIRC2 and BIRC3 are ubiquitin kinases that bind and promote caspase- 3 inactivation and degradation ${ }^{28}$. We validated the expression of cIAP1 by western blotting in HUVEC exposed to UF or DF (Fig. S4c) and found its expression upregulated in UF-exposed HUVEC as expected, correlating with low levels of caspase-3 in UFexposed EC. Treatment of cells with histamine, a pharmacological eNOS activator, that also promotes $\beta$-catenin nuclear translocation ${ }^{15}$, reduced apoptosis in HUVEC exposed to DF (Fig. 5e) and increased the level of survivin (Fig. 6e) suggesting a central role for survivin in regulating cell survival in EC. Together these results support the central role of inhibitors of apoptosis, and their positive regulation of expression by $\beta$-catenin, in mediating the pro-survival effects of the eNOS-cGMP pathway in EC exposed to flow.

\section{Discussion}

Phosphorylation of eNOS on Ser1177 is associated with increased eNOS activity and we show here in static EC that depletion of $\beta$-catenin reduced eNOS phosphorylation on Ser1177 in response to histamine and VEGF stimulation. We also demonstrated increased Ser1177 phosphorylation in EC exposed to UF and found that this was reduced following depletion of $\beta$-catenin. Stabilisation of $\beta$-catenin in HUVEC treated with $\mathrm{LiCl}$ increased Ser1177 phosphorylation in static and flow exposed EC. We conclude that $\beta$-catenin activates eNOS in human EC.

We previously reported that eNOS and $\beta$-catenin interact in static $\mathrm{ECs}^{15}$. We show here that eNOS and $\beta$-catenin co-localise in regions of UF and DF in the mouse aorta in vivo with a higher degree of co-localisation in the inner aortic arch region, supporting our finding that eNOS and $\beta$-catenin show a higher level of interaction in DF-exposed HUVEC in vitro. This may reflect higher eNOS activation under UF conditions and subsequent dissociation of the complex consistent with our finding that acute pharmacological activation of eNOS causes dissociation from $\beta$-catenin ${ }^{15}$. Since reduced levels of $\beta$-catenin associate with reduced eNOS phosphorylation in HUVEC, it may be that interaction of eNOS and $\beta$-catenin facilitates the access or recruitment of kinases that lead to phosphorylation of eNOS and subsequent dissociation of $\beta$-catenin from the complex. PKA and AKT are kinases that phosphorylate Ser1177 and activate eNOS in EC in response to different stimuli such as shear stress or VEGF treatment ${ }^{29}$. PKA and AKT also phosphorylate and stabilise $\beta$-catenin and enhance its transcriptional activity ${ }^{30}$. The identification of the putative kinases recruited by $\beta$-catenin to eNOS will be an important area of further study in order to understand the mechanism of action for the novel $\beta$-catenin-dependent activation of eNOS described here.

We also demonstrate here that $\beta$-catenin and sGC signalling are essential mediators of the pro-survival effects of eNOS in static EC challenged with either TNF $\alpha$ or $\mathrm{H}_{2} \mathrm{O}_{2}$ to induce apoptosis. We previously reported that NO/GMP can stabilise $\beta$-catenin leading to transcriptional changes ${ }^{15}$. Furthermore, for the first time we present evidence showing that both NO-cGMP signalling and $\beta$-catenin transcriptional activity contribute to maintain cell survival in ECs under atheroprone flow conditions. HUVEC treated with the sGC inhibitor ODQ showed an increase in apoptosis in EC exposed to DF that was also observed in both HAEC and HUVEC treated with iCRT5, a specific inhibitor of $\beta$-catenin transcriptional activity. Our findings are supported by Saran et al. ${ }^{31}$ who showed that the pro-apoptotic antagonist of Wnt signalling, sFRP4, causes endothelial dysfunction by suppressing NO-cGMP signalling.

$\mathrm{Wnt} / \beta$-catenin promotes cell survival in static $\mathrm{EC}^{32}$, but to our knowledge this is the first study to show that $\beta$-catenin regulates apoptosis in EC exposed to atheroprone DF. Several groups have studied the pro-survival and anti-apoptotic effects of UF on $\mathrm{EC}^{8,9}$ and identified GSH and NO as key regulators of cell survival in EC under atheroprotective flow conditions. Few studies have assessed the differential expression of the genes and signalling pathways regulating cell survival between disturbed and $\mathrm{UF}^{23,24,33}$. Amini et al. demonstrated that JNK activity/expression was required to drive EC apoptosis at atheroprone sites in mice. Similarly, PERP, a p53 regulator was found to be upregulated in atheroprone areas of the porcine aorta, predisposing EC to apoptosis ${ }^{33}$.

S-nitrosylation of $\beta$-catenin by iNOS can affect junctional permeability ${ }^{34}$, a characteristic usually associated with EC exposed to DF. Recent findings suggest that $\beta$-catenin S-nitrosylation by eNOS impedes binding to the transcription factor TCF4 ${ }^{35}$. This seems to be in contrast with our and others' findings suggesting that $\mathrm{NO}$ favours $\beta$-catenin separation from VE-cadherin and translocation 
Table 1 Apoptosis genes regulated by UF and DF in HUVEC.

\begin{tabular}{|c|c|c|c|c|}
\hline Extrinsic pathway (death receptor) & UF & DF & $p$-value & Fold regulation \\
\hline \multicolumn{5}{|l|}{ Receptors and positive regulators } \\
\hline TNFRSF25 (DR3) & 2.0240 & 0.5587 & 0.0005 & -3.6224 \\
\hline TNFRSF7 (CD27) & 0.0702 & 0.0214 & 0.0098 & -3.2806 \\
\hline TNFRSF6 (FAS) & 1.0081 & 0.4273 & 0.0001 & -2.4041 \\
\hline TNFSF6 (FASLG) & 0.0194 & 0.0095 & 0.0033 & -2.0387 \\
\hline TNFSF5 (CD40LG) & 0.0466 & 0.0237 & 0.0136 & -1.9686 \\
\hline TNFRSF1A (TNFR1) & 6.8953 & 3.7187 & 0.0265 & -1.8542 \\
\hline TNFRSF10B (DR5) & 7.3743 & 4.4220 & 0.0210 & -1.6939 \\
\hline TRAF2 & 0.6532 & 0.4123 & 0.0290 & -1.5841 \\
\hline \multicolumn{5}{|l|}{ Negative regulators } \\
\hline CFLAR (cFLIP/Casper) & 11.0632 & 5.2331 & 0.0005 & -2.1141 \\
\hline IL10 & 0.0046 & 0.0022 & 0.0449 & -2.0572 \\
\hline Intrinsic pathway (mitochondrial/DNA damage) & NT UF & NT DF & $p$-value & Fold regulation \\
\hline \multicolumn{5}{|l|}{ Positive regulators } \\
\hline BCL2L11 & 0.3164 & 0.6653 & 0.0008 & 2.1026 \\
\hline PYCARD & 0.9250 & 1.9230 & 0.0067 & 2.0788 \\
\hline CYCS & 0.2805 & 0.1124 & 0.0003 & -2.4964 \\
\hline APAF1 & 2.1224 & 0.9547 & 0.0032 & -2.2230 \\
\hline BIK & 0.0158 & 0.0075 & 0.0019 & -2.1070 \\
\hline TP53BP2 & 2.2696 & 1.1816 & 0.0059 & -1.9209 \\
\hline AIFM1 & 1.2792 & 0.6753 & 0.0265 & -1.8542 \\
\hline BAK1 & 0.6385 & 0.4079 & 0.0240 & -1.5654 \\
\hline \multicolumn{5}{|l|}{ Negative regulators } \\
\hline BRAF & 0.2838 & 0.1096 & 0.0001 & -2.5910 \\
\hline $\mathrm{BCL} 2 \mathrm{~L} 2$ & 3.6138 & 1.5032 & 0.0006 & -2.4041 \\
\hline BAG1 & 0.1032 & 0.0459 & 0.0281 & -2.2514 \\
\hline MCL1 & 16.5490 & 10.5281 & 0.0263 & -1.5719 \\
\hline Executioners & NT UF & NT DF & $p$-value & Fold regulation \\
\hline NOD1 & 2.3301 & 1.0904 & 0.0001 & -2.1370 \\
\hline Caspase 10 & 2.8597 & 1.5029 & 0.0008 & -1.9028 \\
\hline Caspase 9 & 0.9849 & 0.5731 & 0.0044 & -1.7186 \\
\hline Caspase 8 & 0.4631 & 0.2622 & 0.0067 & -1.7663 \\
\hline CIDEB & 1.5123 & 0.8723 & 0.0228 & -1.7336 \\
\hline ABL1 & 2.3718 & 1.5154 & 0.0207 & -1.5651 \\
\hline \multicolumn{5}{|l|}{ Inhibitors and negative regulators } \\
\hline BIRC1 (NAIP) & 2.4156 & 0.6697 & 0.0015 & -3.6072 \\
\hline BIRC6 & 4.1225 & 1.5979 & 0.0001 & -2.5799 \\
\hline BIRC2 & 5.5505 & 2.7168 & 0.0007 & -2.0430 \\
\hline BIRC5 (Survivin) & 0.1991 & 0.1175 & 0.0210 & -1.6939 \\
\hline NOL3 & 0.8344 & 0.5255 & 0.0061 & -1.5877 \\
\hline
\end{tabular}

Gene names are indicated and common synonyms are shown in brackets. UF and DF represent fold change ( $\left.2^{-\Delta \Delta c t}\right)$ in mRNA expression compared to the housekeeping gene (HPRT1) in non-treated HUVEC exposed to UF or DF for $72 \mathrm{~h}, n=3$. Fold regulation is the negative inverse of the fold change. Fold regulation values greater than one indicate upregulation, and fold regulation values less than one indicate downregulation. In red are indicated genes that can behave like anti or pro-apoptotic depending on alternative splicing. 
to the cell nucleus ${ }^{36}$. In addition to NO, ROS production is necessary for S-nitrosylation in cells ${ }^{37}$. ROS increases in EC exposed to DF leading to low NO bioavailability due to the reaction of ROS with NO to form peroxynitrite that promotes protein S-nitrosylation ${ }^{38}$. S-nitrosylation of $\beta$-catenin may explain the fact that we observe reduced levels of $\beta$-catenin-dependent gene transcription in DF compared to UF-exposed EC. Another interesting possibility that would need further investigation is that Snitrosylation may allow $\beta$-catenin/TCF to discriminate certain promoters or would allow $\beta$-catenin interaction with a different member of the TCF family to activate cell survival vs. proliferation programs (Axin2/ cyclinD1 vs. anti-apoptotic genes).

$\beta$-catenin controls the transcriptional activation of several pro-survival and anti-apoptotic genes such as WISP-1, Bcl-2 and survivin in static cells ${ }^{39,40}$. Survivin has previously been shown to be downregulated in porcine aortic EC exposed to oscillatory flow ${ }^{41}$ and Bcl-2 has been reported to be downregulated under low shear stress compared to high shear stress in HUVEC. Interestingly, the expression of Bcl-2 in EC under high shear stress depends on eNOS activity ${ }^{24}$. Here we show that in HUVEC, survivin and Bcl-2 are upregulated in EC exposed to UF compared to DF, confirming the previous findings ${ }^{24,40}$, and also that their expression is regulated by $\beta$-catenin under flow conditions. In addition, the expression of BIRC3, an inhibitor of apoptosis family member whose expression is flow independent, was also found to be positively regulated by $\beta$-catenin in HUVEC. Survivin has been identified as a key mediator of VEGF ${ }^{40}$ and angiopoietin ${ }^{42,43}$ induced survival; since both these stimuli activate eNOS activity their anti-apoptotic actions may be mediated via an NO-driven increase in $\beta$-catenin activation and consequent increase in the transcription of prosurvival/anti-apoptotic genes. We also observed that survivin expression increased with histamine treatment in HUVEC. The correlation between survivin expression in EC under flow with $\mathrm{NO}$ and $\beta$-catenin levels further supports our finding that $\beta$-catenin is a novel mediator of the pro-survival effects of $\mathrm{NO}$, possibly via regulation of survivin and Bcl-2 expression. Although our experiments have identified clear changes in survivin and Bcl-2 transcript expression, in future work such changes in expression will need confirmation also at the protein level.

Despite the decreased expression of $\mathrm{Bcl}-2$ and survivin in EC exposed to UF following inhibition of $\beta$-catenin, we saw no accompanying increase in apoptosis. This finding suggested that other redundant mechanisms exist in cells exposed to UF that protect the cells from pro-apoptotic stimuli resulting in a powerful pro-survival phenotype. Interestingly we found that the expression of pro-caspase3 was extremely low in HUVEC exposed to UF when compared to DF conditions. Since caspase-3 is considered the main executioner caspase in EC, this may be a parallel regulatory mechanism limiting apoptosis in EC exposed to UF. Caspases are regulated post-translationally by IAPs (inhibitors of apoptosis), a family of E3 ubiquitin ligases. In particular caspase- 3 activity is regulated by BIRC2 (cIAP1) and BIRC3 (cIAP2), that ubiquitinate caspases and promote inactivation and/or subsequent degradation by the proteasome ${ }^{44}$. In HUVEC, cIAP1 which is upregulated by high shear stress was also shown to cause a decrease in caspase-3 activity, although caspase expression was not assessed ${ }^{45}$. Here we describe for the first time that protein levels of caspase- 3 are decreased in EC exposed to atheroprotective UF. In our mRNA apoptosis array, BIRC2 (cIAP1) was upregulated under UF compared to DF and protein expression was confirmed by western blotting, suggesting that high levels of cIAP1 in EC exposed to UF may contribute to the degradation and loss of caspase- 3 in these cells.

Our data suggest a bidirectional regulation of the eNOS- $\beta$-catenin interaction. Upon eNOS activation, $\beta$-catenin translocates to the nucleus to activate gene transcription but $\beta$-catenin also promotes eNOS activation suggesting that activation of one of the pathways will activate the other one providing a cross talk between the Wnt/ $\beta$-catenin and NO. In future work, it would be instructive to also investigate the role of caspases- 8 and -9 , which are upstream of caspase- 3 , as well as the functional relevance of caspase activation, for example, by the use of pharmacological inhibitors, in the apoptosis pathways investigated here. It will also be important to dissect in further detail the effects on eNOS phosphorylation observed in our study, by assaying individual kinases. Nevertheless, our data provide strong evidence of an interaction between two well-established pro-survival pathways and we propose that the eNOS-cGMP$\beta$-catenin axis is essential to maintain cell survival in EC under DF.

\begin{abstract}
Acknowledgements
We thank the Nikon Imaging Center@King's College London (John Harris, Dan Matthews and Isma Ali) for help with microscopy, the Genomics Centre at King's College London for help with qPCR, and Drs. Sarah Chapple and Giovanni Mann in the School of Cardiovascular Medicine \& Sciences at King's College London for providing the mice used in this study. This work was supported by a grant from the British Heart Foundation to AF and CW (PG/15/ 116/31947).
\end{abstract}

\section{Author details}

${ }^{1}$ School of Cardiovascular Medicine \& Sciences, British Heart Foundation Centre of Research Excellence, King's College London, London SE1 9NH, UK. ${ }^{2}$ Department of Bioengineering, Imperial College London, London SW7 2BP, UK. ${ }^{3}$ Department of Comparative Biomedical Sciences, Royal Veterinary College, London NW1 OTU, UK

Conflict of interest

The authors declare that they have no conflict of interest. 


\section{Publisher's note}

Springer Nature remains neutral with regard to jurisdictional claims in published maps and institutional affiliations.

Supplementary Information accompanies this paper at (https://doi.org/ 10.1038/s41419-020-2687-6).

Received: 10 March 2020 Revised: 9 June 2020 Accepted: 15 June 2020 Published online: 30 June 2020

\section{References}

1. Lee, Y. \& Gustafsson, A. B. Role of apoptosis in cardiovascular disease. Apoptosis 14, 536-548 (2009).

2. Tricot, O. et al. Relation between endothelial cell apoptosis and blood flow direction in human atherosclerotic plaques. Circulation 101, 2450-2453 (2000).

3. Bombeli, T., Schwartz, B. R. \& Harlan, J. M. Endothelial cells undergoing apoptosis become proadhesive for nonactivated platelets. Blood 93, 3831-3838 (1999).

4. Cancel, L. M. \& Tarbell, J. M. The role of apoptosis in LDL transport through cultured endothelial cell monolayers. Atherosclerosis 208, 335-341 (2010).

5. Lyons, D. Impairment and restoration of nitric oxide-dependent vasodilation in cardiovascular disease. Int. J. Cardiol. 62(Suppl 2), S101-S109 (1997).

6. Cannon, R. O. III Role of nitric oxide in cardiovascular disease: focus on the endothelium. Clin. Chem. 44, 1809-1819 (1998).

7. Dimmeler, S. \& Zeiher, A. M. Nitric oxide-an endothelial cell survival factor. Cell Death Differ. 6, 964-968 (1999).

8. Dimmeler, S., Haendeler, J., Nehls, M. \& Zeiher, A. M. Suppression of apoptosis by nitric oxide via inhibition of interleukin-1beta-converting enzyme (ICE)-like and cysteine protease protein (CPP)-32-like proteases. J. Exp. Med. 185, 601-607 (1997).

9. Hermann, C., Zeiher, A. M. \& Dimmeler, S. Shear stress inhibits H2O2-induced apoptosis of human endothelial cells by modulation of the glutathione redox cycle and nitric oxide synthase. Arterioscler. Thromb. Vasc. Biol. 17, 3588-3592 (1997).

10. Dimmeler, S., Rippmann, V., Weiland, U., Haendeler, J. \& Zeiher, A. M. Angiotensin II induces apoptosis of human endothelial cells. Protective effect of nitric oxide. Circ. Res. 81, 970-976 (1997).

11. Hoffmann, J. et al. Aging enhances the sensitivity of endothelial cells toward apoptotic stimuli: important role of nitric oxide. Circ. Res. 89, 709-715 (2001).

12. Rossig, L. et al. Nitric oxide inhibits caspase-3 by S-nitrosation in vivo. J. Biol. Chem. 274, 6823-6826 (1999).

13. Rossig, L. et al. Nitric oxide down-regulates MKP-3 mRNA levels: involvement in endothelial cell protection from apoptosis. J. Biol. Chem. 275, 25502-25507 (2000).

14. Hebestreit, $H$. et al. Disruption of fas receptor signaling by nitric oxide in eosinophils. J. Exp. Med. 187, 415-425 (1998).

15. Warboys, C. M. et al. Bidirectional cross-regulation between the endothelial nitric oxide synthase and beta-catenin signalling pathways. Cardiovasc. Res 104, 116-126 (2014).

16. MacDonald, B. T., Tamai, K. \& He, X. Wnt/beta-catenin signaling: components, mechanisms, and diseases. Dev. Cell 17, 9-26 (2009).

17. Ghim, M. et al. Visualization of three pathways for macromolecule transport across cultured endothelium and their modification by flow. Am. J. Physiol. Heart Circ. Physiol. 313, H959-H973 (2017).

18. Warboys, C. M. et al. Disturbed flow promotes endothelial senescence via a p53-dependent pathway. Arterioscler. Thromb. Vasc. Biol. 34, 985-995 (2014).

19. Gavrieli, Y., Sherman, Y. \& Ben-Sasson, S. A. Identification of programmed cell death in situ via specific labeling of nuclear DNA fragmentation. J. Cell Biol. 119, 493-501 (1992).

20. Sangwung, P. et al. KLF2 and KLF4 control endothelial identity and vascular integrity. JCl Insight 2, e91700 (2017).

21. Dardik, A. et al. Differential effects of orbital and laminar shear stress on endothelial cells. J. Vasc. Surg. 41, 869-880 (2005).

22. Sheng, $X$. et al. Effects of FSS on the expression and localization of the core proteins in two Wnt signaling pathways, and their association with ciliogenesis. Int. J. Mol. Med. 42, 1809-1818 (2018).
23. Amini, N. et al. Requirement of JNK1 for endothelial cell injury in atherogenesis. Atherosclerosis 235, 613-618 (2014).

24. Bartling, B. et al. Shear stress-dependent expression of apoptosis-regulating genes in endothelial cells. Biochem. Biophys. Res. Commun. 278, 740-746 (2000).

25. Gonsalves, F. C. et al. An RNAi-based chemical genetic screen identifies three small-molecule inhibitors of the Wnt/wingless signaling pathway. Proc. Natl Acad. Sci. USA 108, 5954-5963 (2011).

26. Handeli, S. \& Simon, J. A. A small-molecule inhibitor of Tcf/beta-catenin signaling down-regulates PPARgamma and PPARdelta activities. Mol. Cancer Ther. 7, 521-529 (2008)

27. Elmore, S. Apoptosis: a review of programmed cell death. Toxicol. Pathol. 35, 495-516 (2007)

28. Vaux, D. L. \& Silke, J. IAPs, RINGs and ubiquitylation. Nat. Rev. Mol. Cell Biol. 6, 287-297 (2005)

29. Heiss, E. H. \& Dirsch, V. M. Regulation of eNOS enzyme activity by posttranslational modification. Curr. Pharm. Des. 20, 3503-3513 (2014).

30. Hino, S., Tanji, C., Nakayama, K. I. \& Kikuchi, A. Phosphorylation of beta-catenin by cyclic AMP-dependent protein kinase stabilizes beta-catenin through inhibition of its ubiquitination. Mol. Cell. Biol. 25, 9063-9072 (2005).

31. Saran, U. et al. SFRP4 signalling of apoptosis and angiostasis uses nitric oxide-cGMP-permeability axis of endothelium. Nitric Oxide 66, 30-42 (2017).

32. Masckauchan, T. N., Shawber, C. J., Funahashi, Y., Li, C. M. \& Kitajewski, J. Wnt/ beta-catenin signaling induces proliferation, survival and interleukin-8 in human endothelial cells. Angiogenesis 8, 43-51 (2005).

33. Serbanovic-Canic, J. et al. Zebrafish model for functional screening of flowresponsive genes. Arterioscler. Thromb. Vasc. Biol. 37, 130-143 (2017).

34. Thibeault, S. et al. S-nitrosylation of beta-catenin by eNOS-derived NO promotes VEGF-induced endothelial cell permeability. Mol. Cell 39, 468-476 (2010).

35. Zhang, Y., Chidiac, R., Delisle, C. \& Gratton, J. P. Endothelial NO synthasedependent S-nitrosylation of beta-catenin prevents its association with TCF4 and inhibits proliferation of endothelial cells stimulated by Wnt3a. Mol. Cell. Biol. 37, e00089-17 (2017).

36. Mei, J. M., Borchert, G. L., Donald, S. P. \& Phang, J. M. Matrix metalloproteinase (s) mediate(s) NO-induced dissociation of beta-catenin from membrane bound E-cadherin and formation of nuclear beta-catenin/LEF-1 complex. Carcinogenesis 23, 2119-2122 (2002).

37. Yang, Y. \& Loscalzo, J. S-nitrosoprotein formation and localization in endothelial cells. Proc. Natl Acad. Sci. USA 102, 117-122 (2005).

38. Hsieh, H. J., Liu, C. A., Huang, B., Tseng, A. H. \& Wang, D. L. Shear-induced endothelial mechanotransduction: the interplay between reactive oxygen species (ROS) and nitric oxide (NO) and the pathophysiological implications. J. Biomed. Sci. 21, 3 (2014)

39. Pennica, D. et al. WISP genes are members of the connective tissue growth factor family that are up-regulated in wnt-1-transformed cells and aberrantly expressed in human colon tumors. Proc. Natl Acad. Sci. USA 95, 14717-14722 (1998).

40. Kaga, S., Zhan, L., Altaf, E. \& Maulik, N. Glycogen synthase kinase-3beta/ beta-catenin promotes angiogenic and anti-apoptotic signaling through the induction of VEGF, BCl-2 and survivin expression in rat ischemic preconditioned myocardium. J. Mol. Cell. Cardiol. 40, 138-147 (2006).

41. Himburg, H. A., Dowd, S. E. \& Friedman, M. H. Frequency-dependent response of the vascular endothelium to pulsatile shear stress. Am. J. Physiol. Heart Circ. Physiol. 293, H645-653 (2007).

42. Papapetropoulos, A. et al. Angiopoietin-1 inhibits endothelial cell apoptosis via the Akt/survivin pathway. J. Biol. Chem. 275, 9102-9105 (2000).

43. Ahmed, A. et al. Angiopoietin-2 confers Atheroprotection in apoE-/mice by inhibiting LDL oxidation via nitric oxide. Circ. Res. 104, 1333-1336 (2009).

44. Choi, Y. E. et al. The E3 ubiquitin ligase CIAP1 binds and ubiquitinates caspase3 and -7 via unique mechanisms at distinct steps in their processing. J. Biol. Chem. 284, 12772-12782 (2009).

45. Jin, X., Mitsumata, M., Yamane, T. \& Yoshida, Y. Induction of human inhibitor of apoptosis protein-2 by shear stress in endothelial cells. FEBS Lett. 529, 286-292 (2002). 